\title{
The Burr-Weibull Power Series Class of Distributions
}

\author{
Broderick O. Oluyede, Precious Mdlongwa, Boikanyo Makubate and Shujiao Huang \\ Georgia Southern University, Botswana International University of Science and Technology, \\ Botswana International University of Science and Technology, BBVA Compass
}

\begin{abstract}
A new generalized class of distributions called the Burr-Weibull Power Series (BWPS) class of distributions is developed and explored. This class of distributions generalizes the Burr power series and Weibull power series classes of distributions, respectively. A special model of the BWPS class of distributions, the new Burr-Weibull Poisson (BWP) distribution is considered and some of its mathematical properties are obtained. The BWP distribution contains several new and well known sub-models, including Burr-Weibull, Burrexponential Poisson, Burr-exponential, Burr-Rayleigh Poisson, Burr-Rayleigh, Burr-Poisson, Burr, Lomax-exponential Poisson, Lomax-Weibull, Lomax-exponential, Lomax-Rayleigh, Lomax-Poisson, Lomax, Weibull, Rayleigh and exponential distributions. Maximum likelihood estimation technique is used to estimate the model parameters followed by a Monte Carlo simulation study. Finally an application of the BWP model to a real data set is presented to illustrate the usefulness of the proposed class of distributions.
\end{abstract}

Keywords: Burr-Weibull distribution, Poisson distribution, Weibull distribution, Burr distribution, maximum likelihood estimation.

\section{Introduction}

The Burr XII (Burr) distribution is a very useful model that was first discussed by Burr (1942) as a two-parameter family. An additional scale parameter was introduced by Tadikamalla (1980). Examples of data modeled by the Burr distribution include household income, crop prices, insurance risk, travel time, flood levels, and failure data. Other applications include simulation, quantal response, approximation of distributions, and development of non-normal control charts. A number of standard theoretical distributions are limiting forms of the Burr distributions. Rodriguez (1977) presented a comprehensive guide to the Burr distribution and showed that this distribution covers various well-known and useful distributions, including the normal, log-normal, gamma, logistic and extreme-value type-I distributions. Morais and BaretoSouza (2011) presented results on a compound class of Weibull and power series distributions. The Burr power series distribution was given by Silva and Cordeiro (2015).

The primary motivation for developing the class of Burr-Weibull Power Series (BWPS) distributions is the versatility and flexibility derived from compounding continuous distributions 
including the new distribution called Burr-Weibull distribution with power series distributions to obtain a new class of distributions with desirable properties including hazard function that exhibits increasing, decreasing, bathtub and upside down bathtub shapes. Another important reason for the development of the BWPS class of distributions is the modeling of income and lifetime data with a model that takes into consideration not only shape and scale but also skewness, kurtosis and tail variation. Also, motivated by various applications of power series distributions in several areas including reliability, exponential tilting (weighting) in finance and actuarial sciences, as well as economics we construct and develop the statistical properties of this new class of generalized compound distribution called the Burr-Weibull Power Series class of distributions and apply it to real lifetime data in order to demonstrate the usefulness of the proposed distribution. This class of distributions generalizes the Burr power series and Weibull power series class of distributions, and their sub-classes, respectively.

The results in this paper are organized in the following manner. In section 2, the Burr-Weibull Power Series (BWPS) distribution is presented. The cumulative distribution function (cdf) of the specific following cases: Burr-Weibull Poisson (BWP), Burr-Weibull geometric (BWG), Burr-Weibull logarithmic (BWL) and Burr-Weibull binomial (BWB) distributions are given. Maximum likelihood estimates of the BWPS model parameters are given in section 3 . The special case of BWP distribution is discussed in section 4. A Monte Carlo simulation study to examine the bias and mean square error of the maximum likelihood estimates for the BWP distribution is also presented in section 4 . Section 5 contains an application of the BWP model to a real data set. A short concluding remark is given in section 6 .

\section{Burr-Weibull power series distribution}

In this section, the Burr-Weibull Power Series (BWPS) class of distributions is presented. In a recent note, Mdlongwa, Oluyede, Amey, and Huang (2017) presented important results on a new distribution called the Burr-modified Weibull (BMW) distribution which includes BurrWeibull (BW) distribution as a special case of BMW when $\lambda=0$. An important motivation for this family of distributions, particularly for use in survival and reliability studies is as follows. Suppose the failure of a device is due to the presence of an unknown number of initial defects of the same kind say $N$, which is identifiable only after causing failure and are repaired perfectly. Let $Y_{i}, i=1, \ldots, N$, denote the time to the failure of the device due to the $i^{\text {th }}$ defect and assume the $Y_{i}$ 's are independent and identically distributed (iid) BW random variables independent of $N$ which is a truncated power series random variable, then the time to the first failure can be modeled by a distribution in the class of BWPS distributions.

The proposed class of distributions can be used for series systems with identical components, which is often the case in many industrial applications and biological organisms. Now, consider a sequence of $N$ iid random variables, say $Y_{i}, i=1, \ldots, N$, from the BW distribution. If $Y$ is a random variable following $\mathrm{BW}$ distribution with parameters $c, k, \alpha, \beta>0$, its cdf is given by

$$
G(y)=1-\left(1+y^{c}\right)^{-k} \exp \left(-\alpha y^{\beta}\right), y \geq 0 .
$$

The corresponding BW survival function and pdf are given by

$$
S(y)=\left(1+y^{c}\right)^{-k} \exp \left(-\alpha y^{\beta}\right), \quad \text { and } \quad g(y)=e^{-\alpha y^{\beta}}\left[1+y^{c}\right]^{-k}\left\{\alpha y^{\beta-1} \beta+\frac{k c y^{c-1}}{\left(1+y^{c}\right)}\right\},
$$

for $c, k, \alpha, \beta>0$, and $y \geq 0$, respectively.

Now, let $N$ be a discrete random following a power series distribution assumed to be truncated at zero, whose probability mass function (pmf) is given by

$$
P(N=n)=\frac{a_{n} \theta^{n}}{C(\theta)}, \quad n=1,2, \ldots,
$$


where $C(\theta)=\sum_{n=1}^{\infty} a_{n} \theta^{n}$ is finite, $\theta>0$, and $\left\{a_{n}\right\}_{n \geq 1}$ a sequence of positive real numbers. The power series family of distributions includes binomial, Poisson, geometric and logarithmic distributions (Johnson, Kotz, and Balakrishnan 1994).

Let $X=Y_{(1)}=\min \left(Y_{1}, \ldots, Y_{N}\right)$. The conditional distribution of $X$ given $N=n$ is given by

$$
G_{X \mid N=n}(x)=1-\prod_{i=1}^{n}(1-G(x))=1-S^{n}(x)=1-\left(1+x^{c}\right)^{-k n} \exp \left(-n \alpha x^{\beta}\right) .
$$

Thus, the cdf of the life length of the whole system, $X$, say $F_{\theta}$, is given by

$$
F_{\theta}(x)=1-\frac{C(\theta S(x))}{C(\theta)}=1-\frac{C\left(\theta\left(1+x^{c}\right)^{-k} \exp \left(-\alpha x^{\beta}\right)\right)}{C(\theta)} .
$$

Note that, $G(t)=\lim _{\theta \rightarrow 0^{+}} F_{\theta}(t)$.

Remark: Let $C^{\prime}(\theta)$ be the derivative of $C(\theta)$, that is, $C^{\prime}(\theta)=\sum_{n=1}^{\infty} n a_{n} \theta^{n-1}$. Then the density of $F_{\theta}$, say $f_{\theta}$, is given by

$$
f_{\theta}(x)=\frac{d F_{\theta}(x)}{d x}=\frac{\theta g(x) C^{\prime}(\theta S(x))}{C(\theta)} .
$$

The hazard and reverse hazard functions are given by

$$
h_{\theta}(x)=\frac{f_{\theta}(x)}{S_{\theta}(x)}=\theta g(x) \frac{C^{\prime}(\theta S(x))}{C(\theta S(x))}, \quad \text { and } \quad \tau_{\theta}(x)=\frac{f_{\theta}(x)}{F_{\theta}(x)}=\theta g(x) \frac{C^{\prime}(\theta S(x))}{C(\theta)-C(\theta S(x))},
$$

respectively, where $S_{\theta}(x)=1-F_{\theta}(x)$. The quantile function of the proposed distribution is obtained by inverting $F_{\theta}(x)=u, 0 \leq u \leq 1$. This is equivalent to solving the equation

$$
-\ln C(\theta S(x))+\ln C(\theta)+\ln (1-u)=0,
$$

which can be done using numerical methods. Consequently, random number can be generated based on equation (4). Some special cases of the BWPS class of distributions are presented in Table 1 for $c, k, \alpha, \beta, \theta>0$.

Table 1: Special Cases of the BWPS

\begin{tabular}{cccc}
\hline Distribution & $a_{n}$ & $C(\theta)$ & cdf \\
\hline Burr-Weibull Poisson & $(n !)^{-1}$ & $e^{\theta}-1$ & $1-\frac{e^{\left(\theta\left(1+x^{c}\right)^{-k} \exp \left(-\alpha x^{\beta}\right)\right.}-1}{e^{\theta}-1}$ \\
Burr-Weibull Geometric & 1 & $\theta(1-\theta)^{-1}$ & $1-\frac{(1-\theta)\left(1+x^{c}\right)^{-k} e^{-\alpha x^{\beta}}}{1-\theta\left(1+x^{c}\right)^{-k} e^{-\alpha x^{\beta}}}$ \\
Burr-Weibull Logarithmic & $n^{-1}$ & $-\log (1-\theta)$ & $1-\frac{\log \left(1-\theta\left(1+x^{c}\right)^{-k} e^{-\alpha x^{\beta}}\right)}{\log (1-\theta)}$ \\
Burr-Weibull Binomial & $\left(\begin{array}{c}m \\
n\end{array}\right)$ & $(1+\theta)^{m}-1$ & $1-\frac{\left(1+\theta\left(1+x^{c}\right)^{-k} e^{-\alpha x^{\beta}}\right)^{m}-1}{(1+\theta)^{m}-1}$
\end{tabular}

The $r^{\text {th }}$ moment of the BWPS distribution is given by

$$
\begin{aligned}
E\left(X^{r}\right) & =\frac{\theta}{C(\theta)} \sum_{n, j=0}^{\infty} \frac{(-1)^{j}(\alpha(n+1))^{j}}{j !} b_{n} \theta^{n}\left[k B\left(k n+k-\left(\frac{r+j \beta}{c}\right), \frac{r+j \beta+c}{c}\right)\right. \\
& \left.+\frac{\alpha \beta}{c} B\left(k n+k-\left(\frac{r+j \beta+\beta}{c}\right), \frac{r+j \beta+\beta}{c}\right)\right],
\end{aligned}
$$


where $B(a, b)=\int_{0}^{1} t^{a-1}(1-t)^{b-1} d t$ is the beta function (see Appendix for the derivation).

\section{Estimation and inference}

Let $X \sim B W P S(c, k, \alpha, \beta, \theta)$ and $\boldsymbol{\Delta}=(c, k, \alpha, \beta, \theta)^{T}$ be the parameter vector. The loglikelihood function $\ell=\ell(\boldsymbol{\Delta})$ based on a random sample of size $n$ is given by

$$
\begin{aligned}
\ell_{n}(\Delta) & =n \ln (\theta)-n \ln (C(\theta))+\sum_{i=1}^{n} \ln \left(g\left(x_{i}\right)+\sum_{i=1}^{n} \ln \left(C^{\prime}\left(\theta S\left(x_{i}\right)\right)\right.\right. \\
& =n \ln (\theta)-n \ln (C(\theta))+\sum_{i=1}^{n}\left(-\alpha x_{i}^{\beta}\right)-(k+1) \sum_{i=1}^{n} \ln \left(1+x_{i}^{c}\right) \\
& +\sum_{i=1}^{n} \ln \left[k c x_{i}^{c-1}+\left(1+x_{i}^{c}\right) \alpha \beta x_{i}^{\beta-1}\right]+\sum_{i=1}^{n} \ln \left(C^{\prime}\left(\theta e^{-\alpha x_{i}^{\beta}}\left(1+x_{i}^{c}\right)^{-k}\right)\right),
\end{aligned}
$$

where the $S(x)$ and $g(x)$ are given in equation (2). The elements of the score vector are given in the Appendix.

The equations obtained by setting the elements of the score vector to zero are not in closed form and the values of the parameters $c, k, \alpha, \beta, \theta$ must be found via iterative methods. The maximum likelihood estimates of the parameters, denoted by $\hat{\boldsymbol{\Delta}}$ is obtained by solving the nonlinear equation $\left(\frac{\partial \ell}{\partial c}, \frac{\partial \ell}{\partial k}, \frac{\partial \ell}{\partial \alpha}, \frac{\partial \ell}{\partial \beta}, \frac{\partial \ell}{\partial \theta}\right)^{T}=\mathbf{0}$, using a numerical method such as Newton-Raphson procedure. The Fisher information matrix is given by $\mathbf{I}(\boldsymbol{\Delta})=\left[\mathbf{I}_{\theta_{i}, \theta_{j}}\right]_{5 X 5}=E\left(-\frac{\partial^{2} \ell}{\partial \theta_{i} \partial \theta_{j}}\right), i, j=$ $1,2,3,4,5$, can be numerically obtained by NLMIXED in SAS or mle 2 package in $\mathrm{R}$ software. The total Fisher information matrix $n \mathbf{I}(\boldsymbol{\Delta})$ can be approximated by

$$
\mathbf{J}_{n}(\hat{\boldsymbol{\Delta}}) \approx\left[-\left.\frac{\partial^{2} \ell}{\partial \theta_{i} \partial \theta_{j}}\right|_{\Delta=\hat{\boldsymbol{\Delta}}}\right]_{5 X 5}, \quad i, j=1, \ldots, 5 .
$$

Note that the expectations in the Fisher Information Matrix (FIM) can be obtained numerically. Let $\hat{\Delta}=(\hat{c}, \hat{k}, \hat{\alpha}, \hat{\beta}, \hat{\theta})$ be the maximum likelihood estimate of $\boldsymbol{\Delta}=(c, k, \alpha, \beta, \theta)$. Under the usual regularity conditions and that the parameters are in the interior of the parameter space, but not on the boundary, we have: $\sqrt{n}(\hat{\boldsymbol{\Delta}}-\boldsymbol{\Delta}) \stackrel{d}{\longrightarrow} N_{5}\left(\underline{\mathbf{0}}, I^{-1}(\boldsymbol{\Delta})\right)$, where $I(\boldsymbol{\Delta})$ is the expected Fisher information matrix. The asymptotic behavior is still valid if $I(\boldsymbol{\Delta})$ is replaced by the observed information matrix evaluated at $\hat{\boldsymbol{\Delta}}$, that is $J(\hat{\boldsymbol{\Delta}})$. The multivariate normal distribution $N_{5}\left(\underline{\mathbf{0}}, J(\hat{\boldsymbol{\Delta}})^{-1}\right)$, where the mean vector $\underline{\mathbf{0}}=(0,0,0,0,0)^{T}$, can be used to construct confidence intervals and confidence regions for the individual model parameters and for the survival and hazard rate functions.

\section{Burr-Weibull Poisson distribution and simulation study}

In this section, we present some results the Burr-Weibull Poisson (BWP) distribution including Monte Carlo simulation study to examine the bias and mean square error of the maximum likelihood estimates. Recall the BWP distribution is a special case of the BWPS class of distributions with $C(\theta)=e^{\theta}-1$ and $a_{n}=\frac{1}{n !}$. The cdf is given by

$$
F_{B W P}(x ; c, k, \alpha, \beta, \theta)=1-\frac{e^{\left(\theta\left(1+x^{c}\right)^{-k} \exp \left(-\alpha x^{\beta}\right)\right)}-1}{e^{\theta}-1},
$$

for $c, k, \alpha, \beta, \theta>0$. 

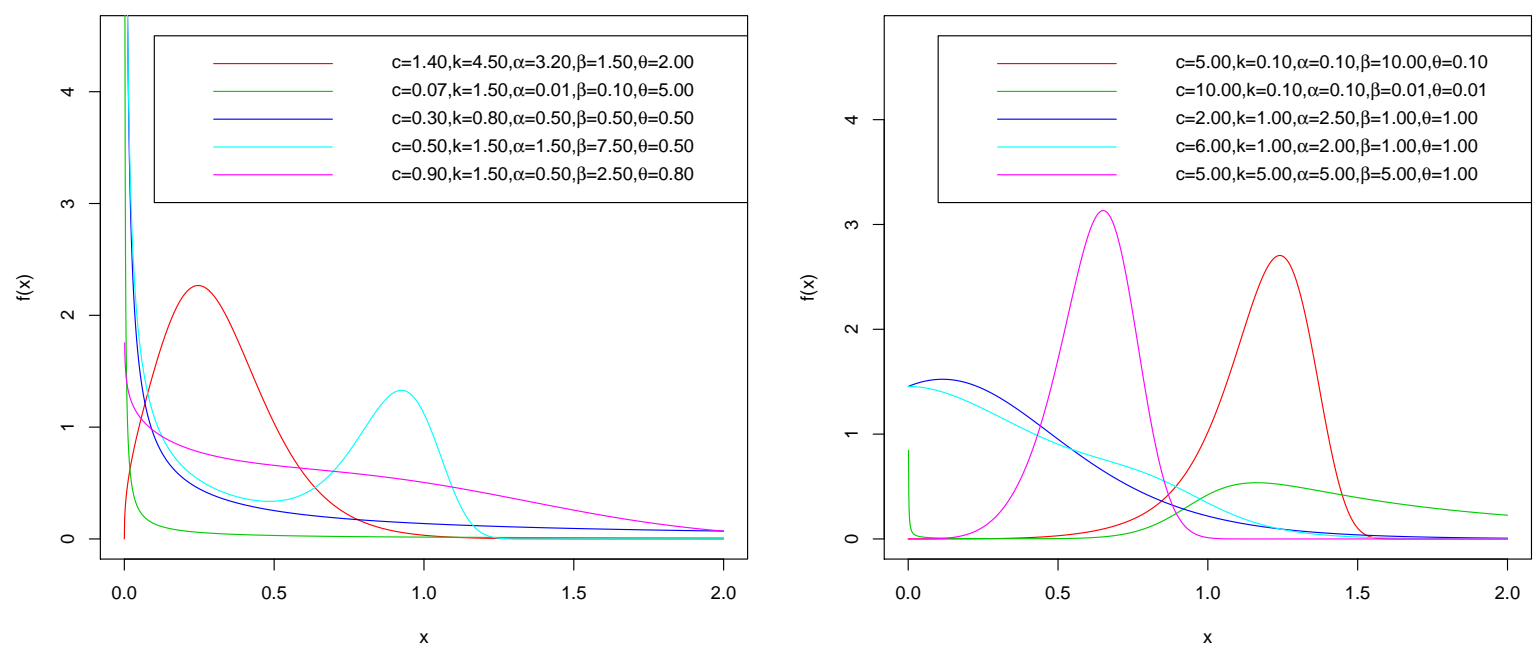

Figure 1: Plots of BWP PDF

Note that when $\beta=1$ in the BWP distribution, we obtain the four parameter Burr XII exponential-Poisson distribution. The plots show that the BWP pdf can be L-shaped, decreasing or right skewed among several other possible shapes as seen in Figures 1.
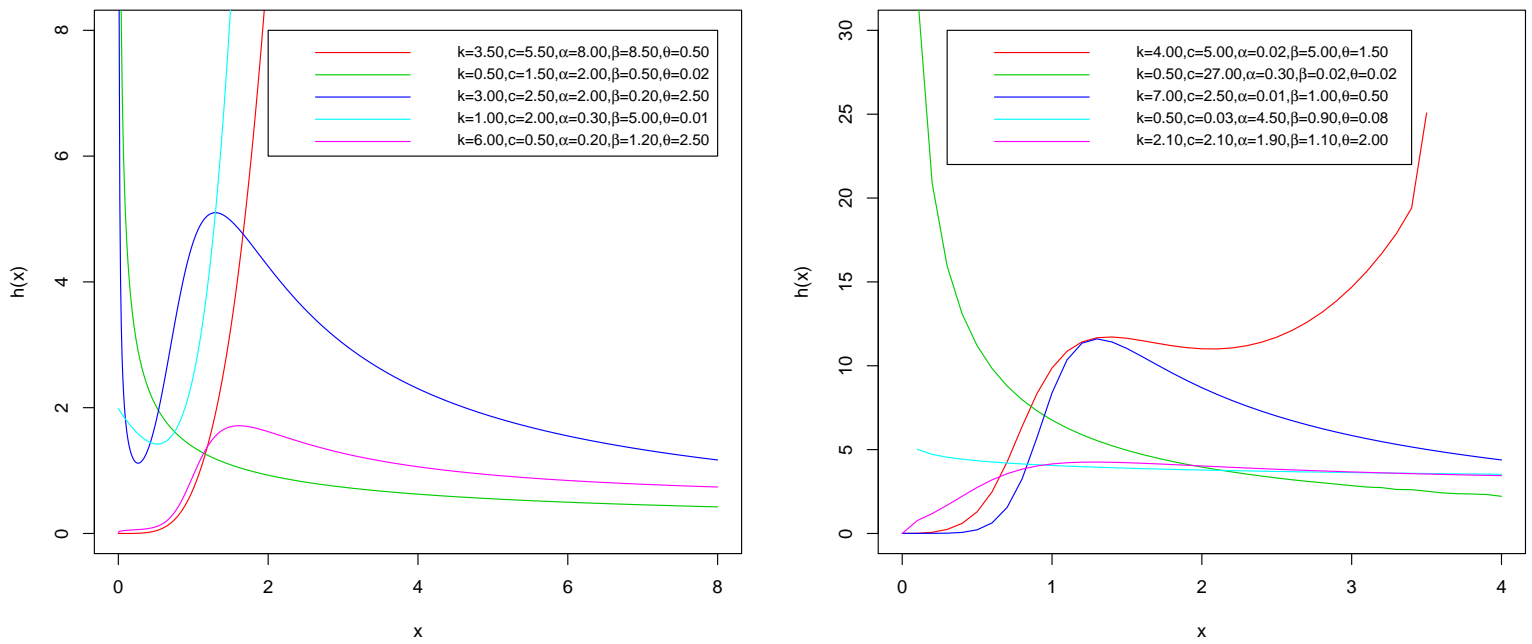

Figure 2: Plots of BWP Hazard Function

Plots of the hazard function are given in Figures 2. The graphs exhibit increasing, decreasing, unimodal, bathtub followed by upside down bathtub, bathtub and upside down bathtub shapes for the selected values of the model parameters. This very attractive flexibility makes the BWP hazard function useful and suitable for non-monotonic empirical hazard behaviors which are more likely to be encountered in practice or real life situations.

\subsection{Sub-models of the BWP distribution}

The BWP distribution contains several new and known sub-models. In this subsection, we present some of the sub-models. 
- If $\beta=1$, we obtain Burr-Exponential Poisson (BEP) distribution.

- If $\beta=2$, we obtain Burr-Rayleigh Poisson (BRP) distribution.

- When $\alpha \rightarrow 0^{+}$, we obtain Burr Poisson (BP) distribution.

- If $c=1$, we obtain Lomax-Weibull Poisson (LWP) distribution.

- If $c=\beta=1$, we obtain Lomax-Exponential Poisson (LEP) distribution.

- If $c=1$ and $\beta=2$, we obtain Lomax-Rayleigh Poisson (LRP) distribution.

- If $c=1$ and $\alpha \rightarrow 0^{+}$, we obtain Lomax-Poisson (LP) distribution.

- When $k \rightarrow 0^{+}$, we obtain Weibull Poisson (WP) distribution.

- If $k \rightarrow 0^{+}$and $\beta=1$, we obtain Exponential-Poisson (EP) distribution.

- If $k \rightarrow 0^{+}$and $\beta=2$, we obtain Rayleigh-Poisson (RP) distribution.

- When $\theta \rightarrow 0^{+}$, we obtain Burr-Weibull (BW) distribution.

- If $\theta \rightarrow 0^{+}$and $\beta=1$, we obtain Burr-Exponential (BE) distribution.

- If $\theta \rightarrow 0^{+}$and $\beta=2$, we obtain Burr-Rayleigh (BR) distribution.

- If $\alpha \rightarrow 0^{+}$and $\theta \rightarrow 0^{+}$, we obtain Burr (B) distribution.

- If $c=1$ and $\theta \rightarrow 0^{+}$, we obtain Lomax-Weibull (LW) distribution.

- If $c=1, \beta=1$ and $\theta \rightarrow 0^{+}$, we obtain Lomax-Exponential (LE) distribution.

- If $c=1, \beta=2$ and $\theta \rightarrow 0^{+}$, we obtain Lomax-Rayleigh (LR) distribution.

- If $c=1, \theta \rightarrow 0^{+}$and $\alpha \rightarrow 0^{+}$, we obtain Lomax (L) distribution.

- If $\theta \rightarrow 0^{+}$and $k \rightarrow 0^{+}$, we obtain Weibull (W) distribution.

- If $\theta \rightarrow 0^{+}, k \rightarrow 0^{+}$and $\beta=2$, we obtain Rayleigh (R) distribution.

- If $\theta \rightarrow 0^{+}, k \rightarrow 0^{+}$and $\beta=1$, we obtain Exponential (E) distribution.

\subsection{Monte Carlo simulation study}

In this section, we examine the performance of maximum likelihood estimates of the BWP model parameters by conducting various simulations for different sizes $(n=35,60,100,200,400,800)$ via the $\mathrm{R}$ package. The true parameter values are set at $I:(c, k, \alpha, \beta, \theta)=(5.5,2.5,0.7,0.5,0.5)$, $I I:(c, k, k, \alpha, \beta, \theta)=(0.5,0.6,0.4,2,1)$ and $I I I:(c, k, \alpha, \beta, \theta)=(5,0.5,1,0.5,0.5)$. We simulate 1000 samples for the true parameters values, also given in the Table 2. Table 2 lists the mean MLEs of the five model parameters along with the respective root mean squared errors (RMSEs). We observe that the estimates approach the true parameter values as the sample size increases, thus implying consistency of the estimates. Also, from the results, we can verify that as the sample size $n$ increases, the mean estimates of the parameters tend to be closer to the true parameter values, since RMSEs decay toward zero. The bias and RMSEs are given by

$$
\operatorname{Bias}(\hat{\theta})=\frac{\sum_{i=1}^{n} \hat{\theta}_{i}}{n}-\theta, \quad \text { and } \quad \operatorname{RMSE}(\hat{\theta})=\sqrt{\frac{\sum_{i=1}^{n}\left(\hat{\theta}_{i}-\theta\right)^{2}}{n}},
$$


respectively.

Table 2: Monte Carlo Simulation Results

\begin{tabular}{|c|c|c|c|c|c|c|c|c|c|c|}
\hline \multirow[b]{2}{*}{ Parameter } & \multirow[b]{2}{*}{ Sample Size } & \multicolumn{3}{|c|}{$(5.5,2.5,0.7,0.5,0.5)$} & \multicolumn{3}{|c|}{$(0.5,0.6,0.4,2,1)$} & \multicolumn{3}{|c|}{$(5,0.5,1,0.5,0.5)$} \\
\hline & & Mean & RMSE & Bias & Mean & RMSE & Bias & Mean & RMSE & Bias \\
\hline \multirow[t]{6}{*}{$\mathrm{c}$} & 35 & 8.4050 & 18.7804 & 2.9050 & 0.5916 & 0.3340 & 0.0916 & 18.5822 & 65.2901 & 13.5822 \\
\hline & 60 & 5.9949 & 1.5954 & 0.4949 & 0.5446 & 0.2266 & 0.0446 & 9.6615 & 20.3314 & 4.6615 \\
\hline & 100 & 5.8853 & 1.1934 & 0.3853 & 0.5207 & 0.1469 & 0.0207 & 10.1759 & 25.6483 & 5.1759 \\
\hline & 200 & 5.6890 & 0.6879 & 0.1890 & 0.5054 & 0.0876 & 0.0054 & 5.8175 & 2.5540 & 0.8175 \\
\hline & 400 & 5.5902 & 0.4483 & 0.0902 & 0.5079 & 0.0645 & 0.0079 & 5.7954 & 2.3297 & 0.7954 \\
\hline & 800 & 5.5254 & 0.3015 & 0.0254 & 0.5198 & 0.0477 & 0.0198 & 5.4040 & 0.9526 & 0.4040 \\
\hline \multirow[t]{6}{*}{$\mathrm{k}$} & 35 & 2.6775 & 0.9934 & 0.1775 & 1.2584 & 1.2832 & 0.6584 & 0.5830 & 0.5268 & 0.0830 \\
\hline & 60 & 2.6260 & 0.5884 & 0.1260 & 1.1109 & 0.9206 & 0.5109 & 0.5058 & 0.2959 & 0.0058 \\
\hline & 100 & 2.5843 & 0.4662 & 0.0843 & 1.0355 & 0.7291 & 0.4355 & 0.4676 & 0.2282 & -0.0324 \\
\hline & 200 & 2.5771 & 0.2966 & 0.0771 & 0.9534 & 0.6013 & 0.3534 & 0.4860 & 0.1597 & -0.0140 \\
\hline & 400 & 2.5126 & 0.2146 & 0.0126 & 0.8742 & 0.4875 & 0.2742 & 0.4503 & 0.1359 & -0.0497 \\
\hline & 800 & 2.5279 & 0.1589 & 0.0279 & 0.7231 & 0.2866 & 0.1231 & 0.4656 & 0.1093 & -0.0344 \\
\hline \multirow[t]{6}{*}{$\alpha$} & 35 & 1.1326 & 0.7176 & 0.4326 & 0.3357 & 0.2953 & -0.0643 & 1.4704 & 0.9665 & 0.4704 \\
\hline & 60 & 1.0625 & 0.5796 & 0.3625 & 0.3612 & 0.2344 & -0.0388 & 1.2684 & 0.5594 & 0.2684 \\
\hline & 100 & 1.0397 & 0.5260 & 0.3397 & 0.3674 & 0.1851 & -0.0326 & 1.2596 & 0.5488 & 0.2596 \\
\hline & 200 & 0.9279 & 0.3792 & 0.2279 & 0.3858 & 0.1288 & -0.0142 & 1.1446 & 0.3024 & 0.1446 \\
\hline & 400 & 0.8385 & 0.2460 & 0.1385 & 0.3776 & 0.0891 & -0.0224 & 1.0620 & 0.1957 & 0.0620 \\
\hline & 800 & 0.8169 & 0.2149 & 0.1169 & 0.3840 & 0.0639 & -0.0160 & 1.0327 & 0.1422 & 0.0327 \\
\hline \multirow[t]{6}{*}{$\beta$} & 35 & 0.5044 & 0.1708 & 0.0044 & 2.8622 & 1.6455 & 0.8622 & 0.4463 & 0.1683 & -0.0537 \\
\hline & 60 & 0.4917 & 0.1202 & -0.0083 & 2.4293 & 0.9872 & 0.4293 & 0.4723 & 0.1115 & -0.0277 \\
\hline & 100 & 0.4994 & 0.0909 & -0.0006 & 2.2547 & 0.6485 & 0.2547 & 0.4887 & 0.0865 & -0.0113 \\
\hline & 200 & 0.4989 & 0.0672 & -0.0011 & 2.0989 & 0.3601 & 0.0989 & 0.5047 & 0.0525 & 0.0047 \\
\hline & 400 & 0.5102 & 0.0484 & 0.0102 & 2.0680 & 0.2161 & 0.0680 & 0.5212 & 0.0437 & 0.0212 \\
\hline & 800 & 0.5106 & 0.0405 & 0.0106 & 2.0422 & 0.1403 & 0.0422 & 0.5205 & 0.0370 & 0.0205 \\
\hline \multirow[t]{6}{*}{$\theta$} & 35 & 1.6513 & 1.6494 & 1.1513 & 7.5261 & 30.3501 & 6.5261 & 5.5386 & 21.2170 & 5.0386 \\
\hline & 60 & 1.5484 & 1.6015 & 1.0484 & 3.0886 & 10.1287 & 2.0886 & 1.5858 & 2.7391 & 1.0858 \\
\hline & 100 & 1.4356 & 1.4497 & 0.9356 & 2.0567 & 1.9375 & 1.0567 & 1.6857 & 6.4734 & 1.1857 \\
\hline & 200 & 1.1507 & 1.0925 & 0.6507 & 1.8855 & 1.6730 & 0.8855 & 0.8811 & 0.7901 & 0.3811 \\
\hline & 400 & 0.8869 & 0.7813 & 0.3869 & 1.6435 & 1.2600 & 0.6435 & 0.5971 & 0.4304 & 0.0971 \\
\hline & 800 & 0.8309 & 0.6704 & 0.3309 & 1.2479 & 0.7948 & 0.2479 & 0.5154 & 0.3532 & 0.0154 \\
\hline
\end{tabular}

\section{Application}

In this section, we present an example to illustrate the flexibility of the BWP distribution and its sub-models for data modeling. BWP distribution is fitted to real data set and these fits are compared to the fits using the sub-models: Burr-Weibull (BW), Burr-exponential Poisson (BEP), Burr-exponential (BE), Burr-Rayleigh Poisson (BRP), Burr-Rayleigh (BR), Burr Poisson (BP), Burr (B), Lomax-Weibull Poisson (LWP), Lomax-Rayleigh Poisson (LRP), Lomax Poisson (LP), Weibull Poisson (WP), and Weibull (W) distributions. We also compare the BWP distribution with the gamma log-logistic Weibull (GLLoGW) (Foya, Oluyede, Fagbamigbe, and Makubate 2017) and beta modified Weibull (BetaMW) (Nadarajah, Cordeiro, and Ortega 2011) distributions. The pdf of the BetaMW distribution is given by

$$
g_{\text {BetaMW }}(x)=\frac{\alpha x^{\gamma-1}(\gamma+\lambda x) \exp (\lambda x)}{B(a, b)} e^{-b \alpha x^{\gamma} \exp (\lambda x)}\left(1-e^{-\alpha x^{\gamma} \exp (\lambda x)}\right)^{a-1}, \quad x>0 .
$$

Also, the pdf of the gamma log-logistic Weibull (GLLoGW) distribution (Foya et al. 2017) is 
given by

$$
\begin{aligned}
g_{\text {GLLoGW }}(x) & =\frac{1}{\Gamma(\delta) \theta^{\delta}}\left(1+x^{c}\right)^{-1} e^{-\alpha x^{\beta}}\left[\left(1+x^{c}\right)^{-1} c x^{c-1}+\alpha \beta x^{\beta-1}\right] \\
& \times\left(-\log \left[1-\left(1+x^{c}\right)^{-1} e^{-\alpha x^{\beta}}\right]\right)^{\delta-1}\left[1-\left(1+x^{c}\right)^{-1} e^{-\alpha x^{\beta}}\right]^{(1 / \theta)-1} .
\end{aligned}
$$

\subsection{Time to failure of kevlar 49/epoxy strands tested at various stress level data}

The data for this example is concerned with the study of the lifetimes of kevlar 49/epoxy spherical pressure vessels that are subjected to a constant sustained pressure until vessel failure, commonly known as static fatigue or stress-rupture. The data set consists of 101 observations of stress-rupture life of kevlar 49/epoxy strands which are subjected to constant sustained pressure at the $90 \%$ stress level until all have failed, so that the complete data set with the exact times of failure is recorded. These failure times in hours, are originally given in Barlow, Towland, and Freeman (1984).

\subsection{Comparison of distributions}

The maximum likelihood estimates (MLEs) of the BWP parameters $\Delta=(c, k, \alpha, \beta, \theta)$ are computed by maximizing the objective function via the subroutine mle 2 in $\mathrm{R}$ ( $\mathrm{R}$ Development Core Team 2011). The initial values for $\mathrm{R}$ code are $c=0.1, k=0.1, \alpha=0.1, \beta=0.1$ and $\theta=5$. In Table 3, the MLEs (standard error in parentheses),-2log-likelihood statistic, Akaike Information Criterion, $A I C=2 p-2 \ln (L)$, Bayesian Information Criterion, $B I C=p \ln (n)-$ $2 \ln (L)$, and Consistent Akaike Information Criterion, $A I C C=A I C+2 \frac{p(p+1)}{n-p-1}$, where $L=L(\hat{\Delta})$ is the value of the likelihood function evaluated at the parameter estimates, $n$ is the number of observations, and $p$ is the number of estimated parameters are presented. The goodnessof-fit statistics $W^{*}$ and $A^{*}$, (Chen and Balakrishnan 1995) are also presented in the table. These statistics can be used to verify which distribution fits better to the data. In general, the smaller the values of $W^{*}$ and $A^{*}$, the better the fit. We also presented the KolmogorovSmirnov (K-S) statistics (and its corresponding p-value) for the purpose of comparisons. The AdequacyModel package in $\mathrm{R}$ was used to evaluate the statistics stated above.

We can use the likelihood ratio test to compare the fit of the BWP distribution with its submodels for a given data set. For example, to test $\beta=1$, the likelihood ratio test statistic is $\omega=2[\ln (L(\hat{c}, \hat{k}, \hat{\alpha}, \hat{\beta}, \hat{\theta}))-\ln (L(\tilde{c}, \tilde{k}, \tilde{\alpha}, 1, \tilde{\theta}))]$, where $\hat{c}, \hat{k}, \hat{\alpha}, \hat{\beta}$ and $\hat{\theta}$ are the unrestricted estimates, and $\tilde{c}, \tilde{k}, \tilde{\alpha}$ and $\tilde{\theta}$ are the restricted estimates. The likelihood ratio test rejects the null hypothesis if $\omega>\chi_{\epsilon}^{2}$, where $\chi_{\epsilon}^{2}$ denote the upper $100 \epsilon \%$ point of the $\chi^{2}$ distribution with 1 degrees of freedom.

For the probability plot, we plotted $F\left(y_{(j)} ; \hat{c}, \hat{k}, \hat{\alpha}, \hat{\beta}, \hat{\theta}\right)$ against $\frac{j-0.375}{n+0.25}, j=1,2, \cdots, n$, where $y_{(j)}$ are the ordered values of the observed data. The measures of closeness, denoted by SS are given by the sum of squares $S S=\sum_{j=1}^{n}\left[F\left(y_{(j)}\right)-\left(\frac{j-0.375}{n+0.25}\right)\right]^{2}$. Plots of the fitted pdf of BWP. BP, B, LEP, GLLOGW and BetaMW models and histogram, observed probability versus predicted probability for the kevlar 49/epoxy strands data are given in Figure 3. 
Table 3: MLEs of the parameters, SEs in parenthesis and the goodness-of-fit statistics for kevlar 49/epoxy failure time data

\begin{tabular}{|c|c|c|c|c|c|c|c|c|c|c|c|c|c|c|}
\hline \multirow[b]{2}{*}{ Model } & \multicolumn{5}{|c|}{ Estimates } & \multicolumn{9}{|c|}{ Statistics } \\
\hline & $c$ & $k$ & $\alpha$ & $\beta$ & $\theta$ & $-2 \log L$ & $A I C$ & $A I C C$ & $B I C$ & $W^{*}$ & $A^{*}$ & $S S$ & $K-S$ & $p$-value \\
\hline BWP & $\begin{array}{c}0.0816 \\
(0.0182)\end{array}$ & $\begin{array}{c}8.0568 \\
(0.3770)\end{array}$ & $\begin{array}{c}0.7810 \\
(0.2609)\end{array}$ & $\begin{array}{c}1.0959 \\
(0.1991)\end{array}$ & $\begin{array}{c}282.9154 \\
(0.0019)\end{array}$ & 202.07 & 212.07 & 212.70 & 225.15 & 0.12 & 0.72 & 0.1134 & 0.0718 & 0.6752 \\
\hline BW & $\begin{array}{c}0.7099 \\
(0.2179)\end{array}$ & $\begin{array}{c}0.4274 \\
(0.6220)\end{array}$ & $\begin{array}{c}0.6842 \\
(0.4694)\end{array}$ & $\begin{array}{c}1.1195 \\
(0.3497)\end{array}$ & $\begin{array}{l}0 \\
-\end{array}$ & 205.72 & 213.72 & 214.35 & 224.18 & 0.15 & 0.91 & 0.1526 & 0.0906 & 0.3778 \\
\hline BEP & $\begin{array}{c}0.6796 \\
(0.1918)\end{array}$ & $\begin{array}{c}0.7718 \\
(0.8200)\end{array}$ & $\begin{array}{c}0.8562 \\
(0.2460)\end{array}$ & $\begin{array}{l}1 \\
-\end{array}$ & $\begin{array}{c}1.2892 \\
(1.3273)\end{array}$ & 204.81 & 212.81 & 213.45 & 223.27 & 0.14 & 0.82 & 0.1304 & 0.0719 & 0.6733 \\
\hline $\mathrm{BE}$ & $\begin{array}{c}0.8910 \\
(0.3950)\end{array}$ & $\begin{array}{c}0.2313 \\
(0.4582)\end{array}$ & $\begin{array}{c}0.8412 \\
(0.2787)\end{array}$ & $\begin{array}{l}1 \\
-\end{array}$ & $\begin{array}{l}0 \\
-\end{array}$ & 206.70 & 212.70 & 213.33 & 220.54 & 0.20 & 1.12 & 0.1867 & 0.0849 & 0.46 \\
\hline $\mathrm{BRP}$ & $\begin{array}{c}0.9010 \\
(0.1557)\end{array}$ & $\begin{array}{c}2.1360 \\
(0.5229)\end{array}$ & $\begin{array}{c}0.0626 \\
(0.0429)\end{array}$ & $\begin{array}{l}2 \\
-\end{array}$ & $\begin{array}{c}1.6216 \\
(1.0473)\end{array}$ & 208.97 & 216.97 & 217.60 & 227.43 & 0.23 & 1.29 & 0.2128 & 0.0911 & 0.3711 \\
\hline $\mathrm{BR}$ & $\begin{array}{c}0.8585 \\
(0.1607)\end{array}$ & $\begin{array}{c}1.3642 \\
(0.2052)\end{array}$ & $\begin{array}{c}0.0821 \\
(0.0400)\end{array}$ & $\begin{array}{l}2 \\
-\end{array}$ & $\begin{array}{l}0 \\
-\end{array}$ & 210.28 & 216.28 & 216.92 & 224.13 & 0.25 & 1.42 & 0.3881 & 0.1314 & 0.0611 \\
\hline BP & $\begin{array}{c}0.9856 \\
(0.1352)\end{array}$ & $\begin{array}{c}2.3855 \\
(0.4024)\end{array}$ & $\begin{array}{l}0 \\
-\end{array}$ & $\begin{array}{l}0 \\
-\end{array}$ & $\begin{array}{c}1.7333 \\
(0.8349)\end{array}$ & 212.40 & 218.40 & 219.04 & 226.25 & 0.34 & 1.87 & 0.3101 & 0.1065 & 0.2019 \\
\hline B & $\begin{array}{c}0.7279 \\
(0.1225)\end{array}$ & $\begin{array}{c}1.6858 \\
(0.1677)\end{array}$ & $\begin{array}{l}0 \\
-\end{array}$ & $\begin{array}{l}0 \\
-\end{array}$ & $\begin{array}{l}0 \\
-\end{array}$ & 216.33 & 220.33 & 220.96 & 225.56 & 0.52 & 2.81 & 1.3549 & 0.2107 & 0.0003 \\
\hline LWP & $\begin{array}{l}1 \\
-\end{array}$ & $\begin{array}{c}1.9773 \\
(0.3246)\end{array}$ & $\begin{array}{c}5.4391 \\
(0.2286)\end{array}$ & $\begin{array}{c}0.0393 \\
(0.0125)\end{array}$ & $\begin{array}{c}382.7459 \\
(0.0006)\end{array}$ & 211.45 & 219.45 & 220.08 & 229.91 & 0.31 & 1.69 & 0.2887 & 0.1077 & 0.1921 \\
\hline LRP & $\begin{array}{l}1 \\
-\end{array}$ & $\begin{array}{c}1.9512 \\
(0.4218)\end{array}$ & $\begin{array}{c}0.0552 \\
(0.0406)\end{array}$ & $\begin{array}{l}2 \\
-\end{array}$ & $\begin{array}{c}1.1729 \\
(0.7172)\end{array}$ & 209.40 & 215.40 & 216.03 & 223.24 & 0.25 & 1.39 & 0.2277 & 0.0912 & 0.3702 \\
\hline LP & $\begin{array}{l}1 \\
-\end{array}$ & $\begin{array}{c}2.3545 \\
(0.2741)\end{array}$ & $\begin{array}{l}0 \\
-\end{array}$ & $\begin{array}{l}0 \\
-\end{array}$ & $\begin{array}{c}1.6666 \\
(0.5421)\end{array}$ & 212.42 & 216.42 & 217.05 & 221.65 & 0.34 & 1.86 & 0.3100 & 0.1060 & 0.2061 \\
\hline WP & $\begin{array}{l}0 \\
- \\
c\end{array}$ & $\begin{array}{l}0 \\
- \\
\alpha\end{array}$ & $\begin{array}{c}1.4042 \\
(0.3730) \\
\beta\end{array}$ & $\begin{array}{c}0.8060 \\
(0.1281) \\
\delta\end{array}$ & $\begin{array}{c}1.2715 \\
(1.1074) \\
\theta\end{array}$ & 204.74 & 210.74 & 211.37 & 218.58 & 0.16 & 0.92 & 0.1507 & 0.0761 & 0.6021 \\
\hline GLLoGW & $\begin{array}{c}0.2365 \\
(0.2965) \\
a\end{array}$ & $\begin{array}{c}0.2591 \\
(0.3727) \\
b\end{array}$ & $\begin{array}{c}0.9648 \\
(0.3741) \\
\alpha\end{array}$ & $\begin{array}{c}4.3962 \\
(10.7190) \\
\gamma\end{array}$ & $\begin{array}{c}0.1396 \\
(0.3332) \\
\lambda\end{array}$ & 204.01 & 214.01 & 214.64 & 227.08 & 0.13 & 0.80 & 0.1293 & 0.0754 & 0.6135 \\
\hline BetaMW & $\begin{array}{c}108.8600 \\
(0.0002)\end{array}$ & $\begin{array}{l}25.6310 \\
(0.0009)\end{array}$ & $\begin{array}{c}1.6632 \\
(0.0279)\end{array}$ & $\begin{array}{c}0.0534 \\
(0.0075)\end{array}$ & $\begin{array}{c}0.0343 \\
(0.0069)\end{array}$ & 207.31 & 217.31 & 217.94 & 230.08 & 0.20 & 1.12 & 0.1915 & 0.0931 & 0.3449 \\
\hline
\end{tabular}

Fitted PDF

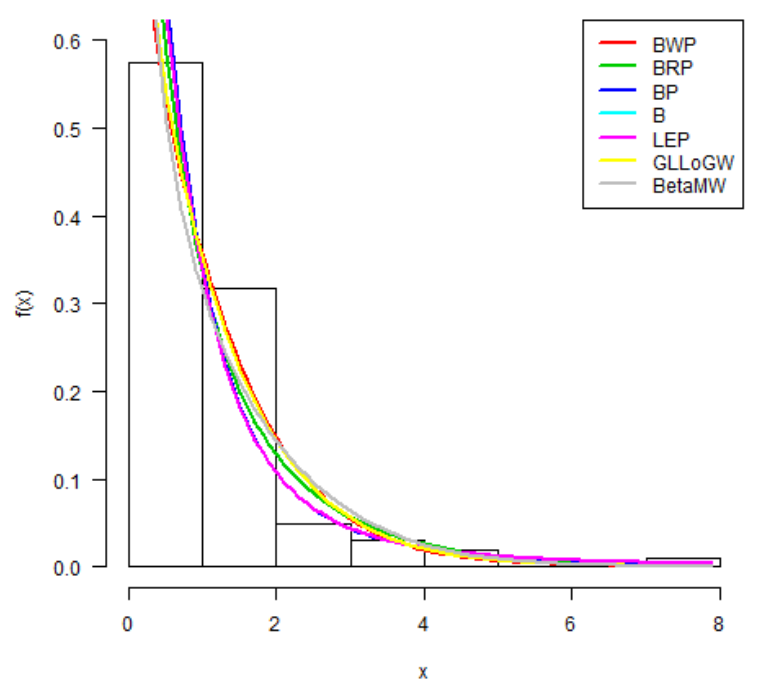

The Graph of Observed vs Expected Probability

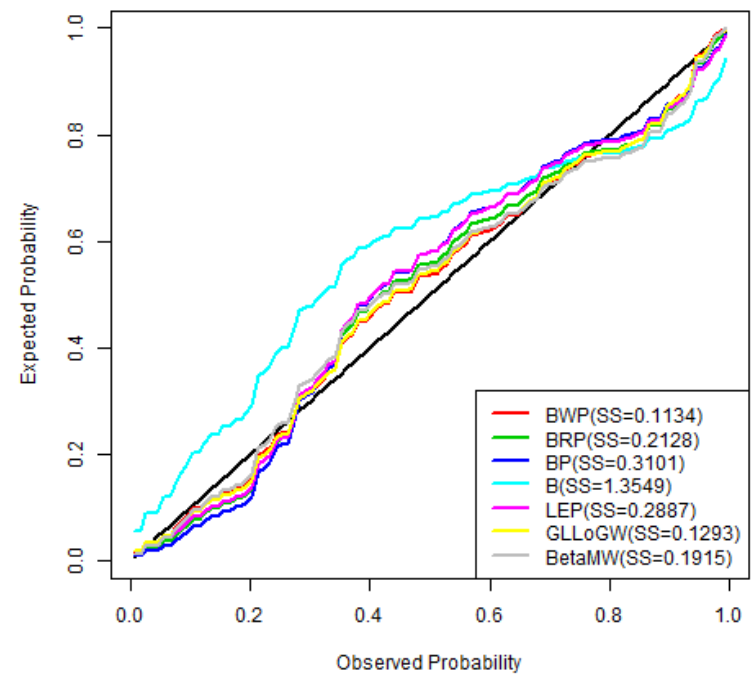

Figure 3: Fitted Densities and Probability Plots for Time to Failure of kevlar 49/epoxy strands tested at various stress level data

The likelihood ratio test statistic for testing $H_{0}$ : BW against $H_{a}$ : BWP and $H_{0}$ : BRP against $H_{a}:$ BWP are $3.65(\mathrm{p}$-value $=0.05607)$ and $6.9(\mathrm{p}$-value $=0.00862)$. We can conclude that there are significant differences in the fit of the BWP and the BW distribution as well as the fit of BWP and BRP distribution. We can also conclude that there are significant differences in the fit of the BWP and the LWP distribution as well as the fit of the BWP and the LP distribution. There are significant differences in the fit of the BWP and the LRP distribution based on the likelihood ratio test. There is no significant difference in the fit of the BWP and 
the WP distribution based on the likelihood ratio test, but the values of the goodness-of-fit statistics $W^{*}$ and $A^{*}$ presented are smallest for the BWP model, an indication that the BWP distribution is the better distribution. The BWP model has the smallest K-S statistic and the largest $\mathrm{p}$-value. The values of the goodness-of-fit statistics $W^{*}$ and $A^{*}$ show that the BWP distribution is by far better than its sub-models and the non-nested GLLoGW and BetaMW distributions. Also, the values of AIC and BIC show that the BWP distribution is better than the non-nested GLLoGW and BetaMW distributions. We conclude that the BWP model has the best fit with respect to the current goodness-of-fit criteria.

\section{Concluding remarks}

A new class of distributions called the Burr-Weibull Power Series (BWPS) distribution have been developed. Estimation of the model parameters via the method of maximum likelihood is presented. The special case of Burr-Weibull Poisson (BWP) distribution is treated in details. A simulation study to assess the performance of the maximum likelihood estimates was conducted. Application of the BWP model to a real data set is presented to illustrate its applicability and usefulness.

\section{Acknowledgements}

The authors are very grateful to the editor and referees for some useful comments on an earlier version of this manuscript which led to this improved version.

\section{Appendix}

The $r^{t h}$ moment of the BWPS distribution is given by

$$
\begin{aligned}
E\left(X^{r}\right) & =\int_{0}^{\infty} x^{r} \frac{\theta g(x) C^{\prime}(\theta S(x))}{C(\theta)} d x \\
& =\frac{\theta}{C(\theta)} \sum_{n=0}^{\infty} b_{n} \theta^{n} \int_{0}^{\infty} x^{r}\left(1+x^{c}\right)^{-k n} e^{-n \alpha x^{\beta}} \\
& \times\left[\left(1+x^{c}\right)^{-k-1} e^{-\alpha x^{\beta}}\left(k c x^{c-1}+\left(1+x^{c}\right) \alpha \beta x^{\beta-1}\right)\right] d x \\
& =\frac{\theta}{C(\theta)} \sum_{n=0}^{\infty} b_{n} \theta^{n} \int_{0}^{\infty} x^{r}\left(1+x^{c}\right)^{-k n-k-1} e^{-(n+1) \alpha x^{\beta}} \\
& \times\left[\left(k c x^{c-1}+\left(1+x^{c}\right) \alpha \beta x^{\beta-1}\right)\right] d x \\
& =\frac{\theta}{C(\theta)} \sum_{n, j=0}^{\infty} \frac{(-1)^{j}(\alpha(n+1))^{j}}{j !} b_{n} \theta^{n} \int_{0}^{\infty} x^{r+j \beta}\left(1+x^{c}\right)^{-k n-k-1} \\
& \times\left[\left(k c x^{c-1}+\left(1+x^{c}\right) \alpha \beta x^{\beta-1}\right)\right] d x \\
& =\frac{\theta}{C(\theta)} \sum_{n, j=0}^{\infty} \frac{(-1)^{j}(\alpha(n+1))^{j}}{j !} b_{n} \theta^{n}\left[k c \int_{0}^{\infty} x^{r+j \beta+c-1}\left(1+x^{c}\right)^{-k n-k-1} d x\right. \\
& \left.+\alpha \beta \int_{0}^{\infty} x^{r+j \beta+\beta-1}\left(1+x^{c}\right)^{-k n-k} d x\right] d x \\
& =\frac{\theta}{C(\theta)} \sum_{n, j=0}^{\infty} \frac{(-1)^{j}(\alpha(n+1))^{j}}{j !} b_{n} \theta^{n}\left[k B\left(k n+k-\left(\frac{r+j \beta}{c}\right), \frac{r+j \beta+c}{c}\right)\right. \\
& \left.+\frac{\alpha \beta}{c} B\left(k n+k-\left(\frac{r+j \beta+\beta}{c}\right), \frac{r+j \beta+\beta}{c}\right)\right],
\end{aligned}
$$


where we have used the transformation $y=\left(1+x^{c}\right)^{-1}$, and $B(a, b)=\int_{0}^{1} t^{a-1}(1-t)^{b-1} d t$ is the beta function.

The elements of the score vector are given by

$$
\begin{aligned}
\frac{\partial \ell}{\partial \theta}= & \frac{n}{\theta}-\frac{C^{\prime}(\theta)}{C(\theta)}+\frac{C^{\prime \prime}\left(\theta\left(e^{-\alpha x_{i}^{\beta}}\left(1+x_{i}^{c}\right)^{-k}\right)\right) e^{-\alpha x_{i}^{\beta}}\left(1+x_{i}^{c}\right)^{-k}}{C^{\prime}\left(\theta\left(e^{-\alpha x_{i}^{\beta}}\left(1+x_{i}^{c}\right)^{-k}\right)\right)} \\
\frac{\partial \ell}{\partial \alpha}= & -\sum_{i=1}^{n} x_{i}^{\beta}+\sum_{i=1}^{n} \frac{\left(1+x_{i}^{c}\right) \beta x_{i}^{\beta-1}}{k c x_{i}^{c-1}+\left(1+x_{i}^{c}\right) \alpha \beta x_{i}^{\beta-1}} \\
& -\sum_{i=1}^{n} \frac{C^{\prime \prime}\left(\theta\left(e^{-\alpha x_{i}^{\beta}}\left(1+x_{i}^{c}\right)^{-k}\right)\right) \theta e^{-\alpha x_{i}^{\beta}}\left(1+x_{i}^{c}\right)^{-k} x_{i}^{\beta}}{C^{\prime}\left(\theta\left(e^{-\alpha x_{i}^{\beta}}\left(1+x_{i}^{c}\right)^{-k}\right)\right)} \\
\frac{\partial \ell}{\partial \beta}= & -\alpha \sum_{i=1}^{n} x_{i}^{\beta} \ln \left(x_{i}\right)+\sum_{i=1}^{n} \frac{\alpha\left(1+x_{i}^{c}\right)\left[x_{i}^{\beta-1}\left(1+\beta \ln \left(x_{i}\right)\right)\right]}{k c x_{i}^{c-1}+\left(1+x_{i}^{c}\right) \alpha \beta x_{i}^{\beta-1}} \\
- & \sum_{i=1}^{n} \frac{C^{\prime \prime}\left(\theta\left(e^{-\alpha x_{i}^{\beta}}\left(1+x_{i}^{c}\right)^{-k}\right)\right) \theta\left(1+x_{i}^{c}\right)^{-k} e^{-\alpha x_{i}^{\beta}} \alpha x_{i}^{\beta} \ln \left(x_{i}\right)}{C^{\prime}\left(\theta\left(e^{-\alpha x_{i}^{\beta}}\left(1+x_{i}^{c}\right)^{-k}\right)\right)} \\
\frac{\partial \ell}{\partial k}= & -\sum_{i=1}^{n} \ln \left(1+x_{i}^{c}\right)+\sum_{i=1}^{n} \frac{c x_{i}^{c-1}}{k c x_{i}^{c-1}+\left(1+x_{i}^{c}\right) \alpha \beta x_{i}^{\beta-1}} \\
- & \sum_{i=1}^{n} \frac{C^{\prime \prime}\left(\theta\left(e^{-\alpha x_{i}^{\beta}}\left(1+x_{i}^{c}\right)^{-k}\right)\right) \theta e^{-\alpha x_{i}^{\beta}}\left(1+x_{i}^{c}\right)^{-k} \ln \left(1+x_{i}^{c}\right)}{C^{\prime}\left(\theta\left(e^{-\alpha x_{i}^{\beta}}\left(1+x_{i}^{c}\right)^{-k}\right)\right)}
\end{aligned}
$$

and

$$
\begin{aligned}
\frac{\partial \ell}{\partial c} & =\sum_{i=1}^{n} \frac{x_{i}^{c} \ln \left(x_{i}\right)}{1+x_{i}^{c}}+\sum_{i=1}^{n} \frac{k\left[x_{i}^{c-1}\left(1+c \ln \left(x_{i}\right)\right)\right]+\alpha \beta x_{i}^{\beta-1}\left(x_{i} \ln \left(x_{i}\right)\right)}{k c x_{i}^{c-1}+\left(1+x_{i}^{c}\right) \alpha \beta x_{i}^{\beta-1}} \\
& -\sum_{i=1}^{n} \frac{C^{\prime \prime}\left(\theta\left(e^{-\alpha x_{i}^{\beta}}\left(1+x_{i}^{c}\right)^{-k}\right)\right) \theta e^{-\alpha x_{i}^{\beta}} k\left(1+x_{i}^{c}\right)^{-k-1} x_{i}^{c} \ln \left(x_{i}\right)}{C^{\prime}\left(\theta\left(e^{-\alpha x_{i}^{\beta}}\left(1+x_{i}^{c}\right)^{-k}\right)\right)} .
\end{aligned}
$$

\section{References}

Barlow RE, Towland RH, Freeman T (1984). "A Bayesian Analysis of Stress-Rupture Life of Kevlar 49/epoxy Spherical Pressure Vessels." Proceedings of the Canadian Conference on Applied Statistics.

Burr IW (1942). "Cumulative Frequency Functions." The Annals of Mathematical Statistics, 13(2), 215-232.

Chen G, Balakrishnan N (1995). "A General Purpose Approximate Goodness-of-fit Test." Journal of Quality Technology, 27(2), 154-161.

Foya S, Oluyede BO, Fagbamigbe AF, Makubate B (2017). "The Gamma Log-logistic Weibull Distribution: Model, Properties and Application." Electronic Journal of Applied Statistical Analysis, 10(1), 206-241. 
Johnson NL, Kotz S, Balakrishnan N (1994). Continuous Univariate Distributions, volume 1. John Wiley \& Sons, New York.

Mdlongwa P, Oluyede BO, Amey A, Huang S (2017). "The Burr XII Modified Weibull Distribution: Model, Properties and Applications." Electronic Journal of Applied Statistical Analysis, 10(1), 118-145.

Morais AL, Bareto-Souza W (2011). "A Compound Class of Weibull and Power Series Distribution." Computational Statistics and Data Analysis, 55(3), 1410-1425.

Nadarajah S, Cordeiro GM, Ortega EMM (2011). "General Results for the Beta-Modified Weibull Distribution." Journal of Statistical Computation and Simulation, 81(10), 12111232 .

R Development Core Team (2011). "R: A Language and Environment for Statistical Computing. R Foundation for Statistical Computing, Vienna, Austria, 2011."

Rodriguez RN (1977). "A Guide to the Burr Type XII Distributions." Biometrika, 64, 129-134.

Silva RB, Cordeiro GM (2015). "The Burr XII Power Series Distribution : A New Compounding Family." Brazilian Journal of Probability and Statistics, 29(3), 565-589.

Tadikamalla PR (1980). "A Look at the Burr and Related Distributions." International Statistical Review, 48(3), 337-344. 


\section{$\mathrm{R}$ algorithms}

The $\mathrm{R}$ codes to compute cdf, pdf, moments, Rényi entropy, mean deviations, maximum likelihood estimates and variance-covariance matrix for the BWPS distribution are available on request from the authors.

\section{Affiliation:}

Broderick O. Oluyede

Department of Mathematical Sciences

Georgia Southern University

Statesboro, GA, 30460, USA

E-mail: boluyede@georgiasouthern.edu

Precious Mdlongwa

Department of Mathematics and Statistical Sciences

Botswana International University of Science and Technology

Palapye, BW

Boikanyo Makubate

Department of Mathematics and Statistical Sciences

Botswana International University of Science and Technology

Palapye, BW

Shujiao Huang

BBVA Compass

Houston, TX, USA

\section{Austrian Journal of Statistics}

published by the Austrian Society of Statistics

Volume 48

January 2019 http://www.ajs.or.at/

http://www.osg.or.at/

Submitted: 2017-01-04 Accepted: 2018-08-28 